\title{
Abstracts from the Eighth European Students Conference of the Charité $\dagger$
}

\author{
Berlin, Germany*
}

\section{THE RESULTS OF BLOOD TESTS TO EVALUATE NONSPECIFIC RESISTANCE IN ADOLESCENTS OF THE CHERCHERSK DISTRICT 10 YEARS AFTER THE CHERNOBYL DISASTER}

\author{
V.V. Kostousova ${ }^{\mathrm{a}}$, N.F. Volkov, Y.Y. Kmw, L.L. Govor \\ ${ }^{a}$ Grodno State Medical Institute, Grodno, Belarus
}

One hundred and ten blood tests were performed on samples from adolescents of Cherchersk (Belarus) and its district where ${ }^{137} \mathrm{Cs}$ territorial contamination, as a result of the Chernobyl disaster, was $15-45 \mathrm{Ci} / \mathrm{km}^{2}$; the morphology and cytochemistry of neutrophils was examined in the samples. The same tests were performed on 28 adolescents from Grodno (control group), where ${ }^{137} \mathrm{Cs}$ contamination was less than 1 $\mathrm{Ci} / \mathrm{km}^{2}$. To analyze the blood, manual blood cell counts, light microscopy of Giemsa stained smears and special stained smears for cytochemical investigations of neutrophils were performed. The mean number of white blood cells was almost the same in both groups (Cherchersk group: $7200 \pm 300 / \mathrm{mm}^{3}$; Grodno control group: $7400 \pm 160 / \mathrm{mm}^{3}$ ). The percentage of neutrophils was decreased in blood from the Cherchersk group $(54.8 \pm 1.3 \%)$ compared with the control group $(60.3 \pm$ $0.90 \%$ ). Also, neutrophils from the Cherchersk group often had morphological abnormalities, notably, hypersegmented nuclei, degenerated (pyknotic) nuclei, toxic granules and vacuoles in the cytoplasm. Cationic protein content was decreased in the Cherchersk group

\footnotetext{
$\dagger$ Abstracts selected by the conference Organizing Committee.

* Held by the Faculty of Medicine (Charité), Humboldt University of Berlin (Berlin, German) on October 15-18, 1997.
}

as compared to the Grodno control group $(100 \pm 2 \mathrm{RU}$ vs. $160 \pm 6 \mathrm{RU}$, respectively), but myelo-peroxidase and cytochrome oxidase content were increased in neutrophils of the investigated group compared with the controls $(250 \pm 3 \mathrm{RU}$ vs $170 \pm 9 \mathrm{RU}$ and $228 \pm 2.4 \mathrm{RU}$ vs $177 \pm 7.2 \mathrm{RU}$, respectively). The following conclusions are made: 1. Increased myeloperoxidase and cytochrome oxidase content may reflect the process of inactivation of bioactive radicals generated by incorporated ${ }^{137} \mathrm{Cs}$. 2 . The tendency of the number of neutrophils to decrease, with frequent morphological abnormalities and lower cationic protein content, may lead to the weakening of counterinfectious defenses or delayed recovery after severe inflammations.

\section{IDENTIFICATION OF THE MORPHOLOGICAL TYPES OF ATHEROSCLEROTIC PLAQUES BASED ON THEIR SPECTRAL CHARACTERISTICS}

\author{
N. Korovine ${ }^{\mathrm{a}}$ \\ a Siberian State Medical University, Tomsk, Russia
}

Presently, different types of lasers for angioplasty are used in clinics around the world. The ultraviolet eximer laser is considered the most progressive in Russia. However, experiments have revealed that this type of radiation is associated with damage to normal vessel tissue which can cause serious postoperative complications. To study the possibility of minimizing these effects, data on vascular wall spectral characteristics in normal and atherosclerotically damaged tissue is necessary. The current study sought to determine reflection and fluorescence spectra of both atherosclerotically damaged and normal human aorta 
walls. Samples of aorta as well as aorta cryostat slices (120-130 um thick) were subjected to spectroscopic examination. The spectrophotometers used were the Specord-M40 and Specol-10. Fluorescent spectrophotometry was also used. The following results were found: 1 . Spectral characteristics of atherosclerotic aorta wall samples were significantly differed from spectral characteristics of normal vascular wall samples. 2. Based on spectral analysis of vascular wall, it is possible to distinguish morphological types of atherosclerotic plaques (lipid or fibron). This will allow for optimization of laser radiation parameters during laser recanalization. Choosing laser energy conditions which induce intensive photochemical processes while causing minimal thermal and stress effects on normal vascular wall will allow effective reconstruction of blood circulation in occluded vessels.

\section{THERMODYNAMIC STUDY OF BIMOLECULAR INTERACTIONS BY MELTING ON OLIGONUCLEOTIDE MICROCHIPS}

\author{
A.V. Fotin ${ }^{\mathrm{a}}$, A.L. Drobyshev ${ }^{\mathrm{b}}$, D.Y. Proudnikov ${ }^{\mathrm{b}}$, \\ A.D. Mirzabekovb \\ a Moscow Institute of Physics and Technology, Moscow, Russia. \\ $\mathrm{b}$ Engelhardt Institute of Molecular Biology, Russian Academy of \\ Sciences. Moscow, Russia.
}

Arrays of oligonucleotides immobilized in gels (oligonucleotide microchips) have found different applications such as, DNA sequencing by hybridization, mutant diagnosis, gene expression analysis and identification of microorganisms. A method of equilibrium melting curve registration and thermodynamic parameter extraction for DNA-DNA duplexes formed by oligonucleotides immobilized in polyacrylamide gel cells and free fluorescently labeled oligonucleotides has been developed. Immobilized duplexes were found to be less stable with respect to those in solution, providing a $0.4 \mathrm{kcal} / \mathrm{mol}$ per base pair decrease in free energy value. We have shown that there exists direct relationship between solution and microchip thermodynamic data for DNA duplexes. Various modifications such as universal bases (5nitroindole), mixtures of natural nucleotides and different linkers have been checked for their influence on stability of the duplexes formed. Discrimination of perfect and mismatch duplexes has been increased by inclusion of terminal universal bases and mixtures. The current authors have managed to significantly narrow melting temperature interval for duplexes of different G-C composition by varying the number of modifications added. Since each microchip element is of a hundred micron size and separates from others, it could be treated as a micro test-tube. Thousands of such test tubes can be arranged on one microchip and be analyzed simultaneously. The technique developed allows one to study a large variety of biomolecular reactions and gives rise to further development in microchip biology.

\section{POSITION REQUIREMENTS FOR PTC MEDIATED mRNA REDUCTION IN BETA- GLOBIN GENES}

\author{
A. Deters ${ }^{\mathrm{a}}$, R. Thermann, G. Neu-Yilik, \\ U. Frede, A.E. Kulozik
}

\footnotetext{
a Humboldt University of Berlin, Labor der Pädiatrischen Molekularbiologie (Charité), Berlin, Germany
}

In beta globin, premature translational termination codons (PTC) are a common molecular basis for betathalassemia, a dyserythropoietic anemia of autosomal recessive inheritance. PTCs, which arise as a consequence of either a frameshift or a nonsense mutation, have been shown to reduce the abundance of mutated mRNA, thus preventing the production of Cterminally truncated protein (nonsense protein). However, if PTCs are located at the 3' end of the penultimate exon or downstream of the last intron, mRNA abundance often reaches wildtype (WT) levels. In this work, the position requirements for PTC mediated mRNA decay in the beta-globin gene has been closely investigated. It has been observed that, unlike PTCs within the first two exons of the beta-globin gene, PTCs in exon III do not induce a reduced abundance of PTC-mutated mRNA. In addition, these exon located PTCs are associated with a more severe phenotype and a dominant mode of inheritance when compared with PTCs within the first two exons. Via site-directed mutagenesis and cloning of a PCR amplified betathalassemic beta globin gene, respectively, human betaglobin genes harboring PTCs at different positions in all of the three exons were generated. To investigate mRNA expression WT or mutant beta-globin genes were transiently transfected into HeLa cells. After northern blotting, the cytoplasmic mRNA was hybridized with a radioactive beta-globin cRNA probe. Hybridization signals were quantified by aligning them to a transfection control signal and comparison with WT mRNA expression. The results show a boundary residing between codon 82 and 88 (exon II) upstream of which PTCs lead to a mRNA reduction and downstream of which PTC containing mRNAs are rescued. According to the presented data, a restriction point at the 3' end of exon II has been defined which separates 
PTCs which reduce abundance of PTC-mutated mRNA from those which do not. Therefore, we conclude that the position of PTCs within the coding region of the beta-globin gene plays an important role in the recognition and reduction of PTC-mutated beta-globin transcripts.

\section{PLASMA-MEDIATED STIMULATION OF SUPEROXIDE ANION AND NITRIC OXIDE PRODUCTION BY POLYMORPHONUCLEAR NEUTROPHILS IN PATIENTS WITH CHEST PAIN}

\author{
M. Balinskia ${ }^{\mathrm{a}}$ T. Rynarzewski
}

a Department of Intensive Therapy, University School of Medical Sciences, Poznan, Poland

During myocardial ischemia, the activation of polymorphonuclear neutrophils results in their adhesion to the endothelium and in the production of free oxygen radicals. Free oxygen radicals increase myocardial injury and superoxide anions $\left(\mathrm{O}^{2-}\right)$, specifically, can inhibit the action of nitric oxide (NO) produced by endothelial cells. Inhibition of NO can result in capillary constriction and enlargement of the ischemic area. It has been suggested that neutrophils also produce NO. The goal of this study was to evaluate plasma-mediated stimulation of superoxide anion and nitric oxide production by polymorphonuclear neutrophils in patients with chest pain. Plasma samples were obtained from 46 emergency patients with chest pain. Patients were divided into two groups by the diagnosis of the attending physicians. The first group consisted of 23 patients (19 men and 4 women, aged 30 - 76 years, average age 52.5) with angina pectoris (AP) and the second group consisted of 23 patients (18 men and 5 women, aged 24 - 55 years, average age 40.2) with non-specific chest pain (NSCP), which includes $82 \%$ intercostal neuralgia, $8 \%$ neurosis, and $8 \%$ gastritis. The plasma samples were evaluated for their ability to stimulate $\mathrm{NO}$ and $\mathrm{O}^{2-}$ production from neutrophils isolated from healthy donors. After incubation of cells with the plasma samples, the production of $\mathrm{NO}$ and $\mathrm{O}^{2-}$ by stimulated and unstimulated neutrophils were analyzed by spectrophotometer. The controls consisted of plasma from healthy donors. There was a significant $(\mathrm{p}<0.05)$ increase in incubated neutrophil $\mathrm{O}^{2-}$ production from the plasma of AP patients $(16.0 \pm 4.2$ nmol/5 x 106/30 minutes) in comparison to NSCP patients $(12.3 \pm 3.3)$. No differences were observed in plasma stimulation of NO production by neutrophils (4.4 $\pm 1,0,5.21 \pm 0,9 \mathrm{mmol} / 20 \times 106 / \mathrm{h}$ respectively).
After incubation with plasma samples, zymosanstimulated neutrophils showed similar ability to produce $\mathrm{O}^{2-}(47.3 \pm 2.6 ; 41.8 \pm 2.8$ respectively) and NO $(24.8 \pm 4.0,24.6 \pm 1.9$ respectively). The current work shows that during episodes of angina pectoris, mediators stimulating neutrophils are released resulting in the production of $\mathrm{O}^{2-}$ by the neutrophils. It has not been proven that NO production was stimulated by plasma from angina pectoris patients.

\section{CHARACTERIZATION OF A CHEMOTHERAPY-INDUCIBLE VECTOR SYSTEM AND STRATEGIES FOR MODIFICATION OF PROMOTER- INDUCIBILITY}

\author{
J. Wendt ${ }^{\mathrm{a}}$, W. Walther, U. Stein, P.M. Schlag
}

\begin{abstract}
${ }^{a}$ Humboldt University of Berlin, FG Chirurgie/ Chirurgische Onkologie, Max Delbrück Centrum für Molekulare Medizin, Berlin, Germany
\end{abstract}

Conditionally active promoters in vector systems can be used to effectively regulate the expression of therapeutically relevant genes in tumor cells. Numerous studies have shown that the promoter of the multidrug resistance gene (mdr1) is induced by several lipophilic agents called multidrug resistance (MDR)related drugs. The current authors have characterized a vector system using a mutated mdr1-promoter in order to regulate the expression of therapeutic genes, such as the tumor necrosis factor alpha (TNF $\alpha$ ) gene, in tumor cells. Stably transduced human mammary and colon carcinoma cells were treated with MDR-related drugs. Inducibility of promoter-driven TNF $\alpha$ expression was analyzed at the level of mRNA and protein. It was shown that gene expression is inducible by the MDRassociated drugs adriamycin, vincristine, etoposide and taxol. For instance, the treatment of transduced cells with adriamycin increased the TNF $\alpha$-expression up to 13 -fold. However, TNF $\alpha$ expression was not induced by 5-fluorouracil, which is a non-MDR-associated drug. These results clearly demonstrate the inducibility of the new cassette vector system by chemotherapeutic agents. However, for efficient therapeutic effects the induction potential will need to be increased. Our strategies for modification of promoter-inducibility include multimerization of $\mathrm{mdr} 1$ promoter regions containing drug-responsive elements. The objective is to construct a vector with low basal expression of a therapeutic gene and a high induction-capacity by chemotherapy directed towards the characterized mdr1 promotor-sequences. 


\section{POST PARTUM DEPRESSION: LEVELS AND ASSOCIATED RISK FACTORS IN URBAN PAKISTANI WOMEN.}

\author{
A.H. Haider ${ }^{\text {a }}$, A. Shaukat and A. Faizi \\ a The Aga Khan University Medical College, Karachi, Pakistan
}

The current study aimed to quantify the burden of Post Partum Depression (PPD) in urban Pakistani women and to identify its etiologic/risk factors. A prospective cohort study with a nested case control was used. Exposure was defined as giving birth to a live child during the past six to eight weeks. The unexposed group consisted of mothers who had their last pregnancy between the last two to five years. The setting was the the Aga Khan University Hospital's Community Health Center - Immunization Clinic. Two trained female interviewers administered, to 61 women in each group (122 in total), a standardized questionnaire which contained the Urdu version of the validated Hospital Anxiety Depression Scale. Socioeconomic data and some specific questions were administered only to the post partum group. It was found that $6.6 \%$ of the post partum group were suffering from PPD. In the unexposed group the level of depression stood at $5 \%$. Known risk factors in the west such as non-cohabitation of a spouse, lack of insurance, a past history of psychiatric disorders, alcoholism and the use of oral contraceptive pills were hardly found in our group of post partum women. Quantifiable measures of social support, such as the presence of a husband, adequate time to rest and a responsible person to take care of the child, were present in more than $95 \%$ of the post partum women interviewed. It is concluded that in the current setting post partum females are not at a significantly higher risk of developing depression. This finding is attributed to the unique sociodynamics of Pakistani society which plays a protective role against post partum depression.

\section{EVALUATION OF CYFRA 21-1 AND CEA AS BLOOD TUMOR MARKERS IN PATIENTS WITH NON-SMALL-CELL LUNG CANCER}

\section{R. Petar ${ }^{\mathrm{a}}$, P. Natasha}

a School of Medicine at University "St. Cyrill and Methodius", Skopje, Republic of Macedonia

Over the last few years, several serum components have been confirmed as lung cancer markers. One of these is cytokeratin 19, a member of the intermediate filament family, which is overexpressed in lung carcinoma. One of its fragments, CYFRA 21-1, is released in the bloodstream upon cell death. Two monoclonal antibodies, BM 19-1 21 and KS 19-1, reactive to different epitopes on cytokeratin 19 were used in an immunoradiometric assay to measure levels of cytokeratin 19 fragments in serum samples. Meanwhile, carcinoembryonic antigen (CEA) is mostly secreted by digestive tract cancers and their metastases, but is also found in other types of cancer, such as lung breast carcinoma. The serum level of CYFRA 21-1 was evaluated in comparison with that of CEA in 26 patients with histologically verified non-small-cell lung cancer and in 19 patients with benign lung diseases. Patients with chronic renal failure and benign digestive inflammatory diseases were excluded. After setting cutoff levels of $3.3 \mathrm{ng} / \mathrm{ml}$ for CYFRA $21-1$ and $5 \mathrm{ng} / \mathrm{ml}$ for CEA, it was determined that: CYFRA 21-1 (postulated specificity of 90\%) showed highest sensitivity for squamous cell lung cancer (66\%) and CEA (specificity of $84 \%$ ) showed greatest sensitivity for adenocarcinoma (63\%). Specificities were determined against benign lung diseases. The present study suggests that CYFRA and CEA serum levels correlate with cancer burden and may contribute to detection of metastases.

\section{THE BILGINTURAN'S SYNDROME: A MONOGENIC FORM OF SEVERE HYPERTENSION ASSOCIATED WITH BRACHYDACTYLY WHICH RESEMBLES ESSENTIAL HYPERTENSION AND MAPS TO HUMAN CHROMOSOME 12P}

\author{
H.R. Toka ${ }^{a}$, S. Baehring, A. Aydin, O. Toka, T.F. \\ Wienker, H. Schuster and F.C. Luft
}

a Humboldt University of Berlin, Berlin, Germany

In 1973, Bilginturan et al. described a unique Turkish family with autosomal dominant inherited hypertension associated with brachydactyly type E. The life expectancy of the affected family members was clearly reduced and affected relatives died, almost without exception, by stroke before the age of 50. Hypertension cosegregated at a rate of $100 \%$ with brachydactyly. Sixty-six family members were reinvestigated under genetic field working conditions at the Black Sea coast of Turkey. Six family members, one non affected and five affected relatives were examined in the clinical research unit at Humboldt University (Berlin, Germany). Affected persons are not salt-sensitive. The elevated blood pressure resembles essential hypertension. MRI studies performed in 29 family members show neurovascular anomalies at the left ventrolateral medulla in all affected subjects. This 
finding could be a primary, a secondary or an independent phenomenon. With microsatellite markers and linkage analysis, the gene locus causing hypertension and brachydactyly was mapped on chromosome 12p. Different strategies to reduce the gene locus ( $3 \mathrm{cM}$ distance between the cosegregating flanking markers) and identify the gene(s) are currently being performed. One strategy is comparison of the gene locus with a deleted segment on chromosome $12 \mathrm{p}$ in a Japanese boy with brachydactyly. Another strategy is comparison of two additional families with the same syndrome.

\section{BIOLOGICAL AND SOCIAL DIFFERENTIATION OF KALENJIN SPEAKING KENYAN RUNNERS}

\author{
M. Nurok ${ }^{a}$, A.G. Morris ${ }^{b}$ and T.D. Noakes ${ }^{a}$ \\ a UCT/MRC Bioenergetics of Exercise Research Unit, Sports \\ Science, Institute of South Africa, Newlands, Cape Town, South \\ Africa \\ b Department of Anatomy, UCT Medical School, Anzio Road, \\ Observatory, Cape Town, South Africa.
}

Kenyan runners currently dominate the international long and middle distance running arena. The overwhelming majority of Kenyan runners come from the Kalenjin speaking people who comprise a minority of the Kenyan population. There has been anecdotal evidence to suggest the presence of specific lineages with superior running ability within Kenyas Kalenjin speaking population. The present case-control study aimed to establish any biological or socio-cultural differences between a group of runners $(n=25)$, and non-runners $(\mathrm{n}=25)$ attending St. Patricks School (Inten, Kenya), in the heart of Kenyas Kalenjin speaking region. A standard questionnaire was administered by a headmaster at the school, detailing biological and socio-cultural data including specific information regarding biological relatives. No significant difference in tribal background was found between the samples (almost exclusively Kalenjin) when traced to maternal and paternal grandparents. Eighteen runners were found to have at least one first degree relative running competitively, compared to two non-runners. Other significant findings included: 24 non-runners intended to pursue post school education as compared to 10 runners, when controlled for the six runners intending to become professional athletes the difference remained significant; four runners competing at a provincial level or above intended to pursue a career in athletics as compared to two runners below this level; the mean age, weight and height of the running sample was greater (by 0.74 of a year, $5.4 \mathrm{~kg}$, and 0.09 meters respectively); the mean standing long jump, and $\mathrm{cm}$ jumped per kg body weight was greater for the runners. No significant differences were found between Body Mass Indices of runners and non-runners, or in their numbers of parents with post school education. These findings are in keeping with the suggestion that specific runner families exist within the Kalenjin population. 